Filomat 27:8 (2013), 1455-1461

DOI 10.2298/FIL1308455E
Published by Faculty of Sciences and Mathematics, University of Niš, Serbia

Available at: http://www.pmf.ni.ac.rs/filomat

\title{
Some consequences of an inequality on the spectral multiplicity of graphs
}

\author{
Aleksandra Erića ${ }^{a}$ C.M. da Fonseca ${ }^{b}$ \\ ${ }^{a}$ Faculty of Civil Engineering, University of Belgrade, 11000 Belgrade, Serbia \\ ${ }^{b}$ Departamento de Matemática, Universidade de Coimbra, 3001-501 Coimbra, Portugal
}

\begin{abstract}
We present two distinct applications of an inequality relating the multiplicity of an eigenvalue of a graph to a certain subgraph. The first is related to a recent classification, established by Kim and Shader, for the class of those trees for which each of the associated matrices have distinct eigenvalues whenever the diagonal entries are distinct. We analyze the minimum number of distinct diagonal entries and the corresponding location, in order to preserve such multiplicity characterization. The second application involves a new property of a star set of a graph due to P. Rowlinson.
\end{abstract}

\section{Preliminaries}

For a given $n \times n$ real symmetric matrix $A=\left(a_{i j}\right)$, we define the graph of $A$, and write $G(A)$, as the undirected graph whose vertex set is $\{1, \ldots, n\}$ and edge set is $\left\{i j \mid i \neq j\right.$ and $\left.a_{i j} \neq 0\right\}$. On the other hand, for a given (weighted) graph $G$, we may define $A(G)=\left(a_{i j}\right)$ to be the (real) symmetric matrix whose graph $G(A)$ is $G$. We devote our attention to the set

$$
\mathcal{S}(G)=\left\{A \in \mathbb{R}^{n \times n} \mid A \text { is symmetric and } G(A)=G\right\},
$$

i.e., the set of all symmetric matrices sharing a common graph $G$ on $n$ vertices. Nevertheless, all results can easily be extended to complex Hermitian matrices.

If $G$ is a tree, then the matrix $A(G)$ is called acyclic. In particular, if $G$ is a path, we order the vertices of $G$ such that $A(G)$ is a tridiagonal matrix.

We will often omit the mention of the graph of the matrix if it is clear from the context.

Let us denote the (algebraic) multiplicity of the eigenvalue $\theta$ of a symmetric matrix $A=A(G)$ by $m_{A}(\theta)$. The $(n-1) \times(n-1)$ principal submatrix, formed by the deletion of row and column indexed by $i$, which is equivalent to removing the vertex $i$ from $G$, is designated by $A(G \backslash i)$.

As a consequence of Cauchy's Interlacing Theorem for the eigenvalues of symmetric matrices, one can deduce that

$$
m_{A(G)}(\theta)-1 \leqslant m_{A(G \backslash i)}(\theta) \leqslant m_{A(G)}(\theta)+1
$$

\footnotetext{
2010 Mathematics Subject Classification. Primary 15A18; Secondary 15A48, 05C50. Keywords. Graph eigenvalue; Tree; Double star; Acyclic matrix; Star complement. Received: 9 July 2012; Accepted: 30 May 2013

Communicated by Dragan Stevanović

Email addresses: eric@grf.rs (Aleksandra Erić), cmf@mat.uc.pt (C.M. da Fonseca)
} 
In the case that $m_{A(G \backslash i)}(\theta)=m_{A(G)}(\theta)+1$, the vertex $i$ was designated by Jonhson et al. as a Parter-vertex of $A$ for $\theta[13,15]$, motivated by the work of Seymour V. Parter [18], complemented and extended by Gerry $\mathrm{M}$. Wiener in [21] on the location and multiplicity of eigenvalues of sign symmetric acyclic matrices. Note that this concept has also been considered by Godsil in the context of the matching polynomial theory as a $\theta$-positive vertex of $G$ [10-12]. In the case that $m_{A(G)}(\theta) \leqslant m_{A(G) i}(\theta), i$ is called a Fiedler-vertex of $A$. In [17] Kim and Shader provide geometric characterizations of Fiedler- and Parter-vertices of acyclic matrices, and give a geometric proof for the so-called Parter-Wiener Theorem.

Theorem 1.1 (Parter-Wiener Theorem, [13]). Let $A$ be an (irreducible) acyclic matrix, and let $\lambda$ be an eigenvalue of $A$ with $m_{A(G)}(\lambda) \geqslant 2$. Then there exists a Parter-vertex i of $A$ for $\lambda$ such that $\lambda$ is an eigenvalue of at least three of the direct summands of $A(G \backslash i)$.

Moreover, they describe the structure of an acyclic matrix in terms of Fiedler- and Parter-vertices enabling the construction an acyclic matrix of a desired form according to the locations of those vertices.

We remark that Theorem 1.1 was reformulated in the survey work [6], motivated by some of the seminal papers on matching polynomials due to Godsil (cf., e.g., [10-12]).

Later, in 2008, Kim and Shader [16] introduced the subset of $\mathcal{S}(G)$, having distinct diagonal entries,

$$
\mathcal{S D}(G)=\left\{A=\left(a_{i j}\right) \in \mathcal{S}(G) \mid a_{k k} \neq a_{\ell \ell}, \text { for } k \neq \ell\right\},
$$

and classified all the trees $T$ such that every matrix in $\mathcal{S D}(T)$ has only simple eigenvalues.

Theorem 1.2 ([16]). Let $T$ be a tree that is not a path. Then each matrix in $\mathcal{S D}(T)$ has distinct eigenvalues if and only if each vertex of degree 3 or greater in $T$ has at most one branch which is not a pendant vertex.

The proof for the sufficiency of Theorem 1.2 is based on the Parter-Wiener Theorem while the necessity is a constructive procedure using the following lemma.

Lemma 1.3 ([16]). Let $T$ be a nontrivial tree. Then there exists a singular matrix in $\mathcal{S D}(T)$ with all nonzero diagonal entries.

We now define the major family of acyclic graphs that we will consider.

Definition 1.4. A double star is the tree obtained from two vertex disjoint stars by connecting their centers by a path.

The path of previous definition can be, eventually, trivial, i.e., reduced to a single vertex. In this sense, a star is itself a double star. A path is a double star as well. For the sequel we fix the following notation: if the two pendant stars have sizes $n_{1}$ and $n_{2}$, and the path has size $p$, then the double star is notated by $S_{n_{1} n_{2}}^{p}$. Therefore $S_{n_{1} n_{2}}^{1}$ is a star with size $n_{1}+n_{2}-1$, and $S_{00}^{p}$ is a path of size $p$. Observe that $S_{n_{1} 0}^{p}$ has size $n_{1}+p-1$, and, otherwise, $S_{n_{1} n_{2}}^{p}$ has size $n_{1}+p+n_{2}-2$.

The main graph characterization of $\mathcal{S D}(T)$ is a consequence of Theorem 1.2.

Corollary 1.5 ([16]). Let $T$ be a tree. Then each matrix in $\mathcal{S D}(T)$ has distinct eigenvalues if and only if $T$ is a double star.

Recently Johnson et al. [14] characterized all graphs $G$ such that any real symmetric matrix that has graph $G$ has no eigenvalues of multiplicity greater than 2 . 


\section{Some results on multiplicities}

Following a similar procedure [16], but avoiding Theorem 1.1, we start by showing that the family of double stars characterizes a broader class of symmetric matrices. We will determine the minimum number of distinct diagonal entries of such matrices having all eigenvalues simple. In this section, we recall some results. The reader is referred to [1] for a full account regarding the terminology used throughout.

Let $G$ be a graph and let $A=A(G) \in \mathcal{S}(G)$. We denote by $\varphi(A(G), x)$ the characteristic polynomial of $A$. Motivated by the results on matching polynomials [10, 11], the second author showed in [9]:

Proposition 2.1. Let $i$ and $j$ be two adjacent vertices in a tree $T$ such that $\varphi(A(T \backslash i), x)$ and $\varphi(A(T \backslash i j), x)$ have no common zero. Then $\varphi(A(T), x)$ and $\varphi(A(T \backslash i), x)$ also have no common zero.

Using the interlacing property and Proposition 2.1, we may also conclude that the eigenvalues of $A(T)$ (and of $A(T \backslash i)$ ) are all simple. The induction provides the following proposition.

Proposition 2.2. Suppose that $T$ is a tree resulting from joining a tree $H$ and a path at one of its pendant vertices, say $i$. If $\varphi(A(H), x)$ and $\varphi(A(H \backslash i), x)$ have no common root, then all eigenvalues of $A(T)$ are simple.

We observe that there is a disfiguring misprint contained in the original result [9, Corollary 7.4.].

In a slight extension of the main result of [8], the second author stated the following lemma.

Lemma 2.3 ([5]). Let $P$ be a path which does not contain any edge of any cycle in graph $G$. Then

$$
m_{A(G \backslash P)}(\theta) \geqslant m_{A(G)}(\theta)-1 .
$$

This result has many interesting implications on spectral graph theory still to be explored. In particular, we have the following lemma for trees of which we will discuss some consequences in the next sections.

Lemma 2.4 ([8]). Let $P$ be a path in a tree $T$ and $A(T) \in \mathcal{S}(T)$. If $\theta$ is an eigenvalue of $A(T)$, then

$$
m_{A(T \backslash P)}(\theta) \geqslant m_{A(T)}(\theta)-1 \text {. }
$$

In the two final sections we discuss some other interesting consequences.

\section{Simple multiplicities on stars}

The classical result asserting that the eigenvalues of any irreducible tridiagonal matrix are real and distinct is a well known fact from, for example, the theory of orthogonal polynomials (cf. [20, Theorem 3.3.1]). One can easily deduce this fact from Proposition 2.1 or Proposition 2.2 as well. We also observe that this property does not depend on the distinctness of diagonal entries. In the sequel, we will prove a more general result among all double stars, providing a deep insight of the mentioned property. But first let us begin with the particular case of a star, and with a simple but insightful observation.

Lemma 3.1. Let $T$ be a star of order $n$ with central vertex labeled by 1 . Suppose that $A=\left(a_{i j}\right)$ is in $\mathcal{S}(T)$, with $a_{22}, a_{33}, \ldots, a_{n n}$ distinct. Then $a_{i i}$, for $i \geqslant 2$, is not an eigenvalue $A$.

Proof. Under the conditions of the lemma, $a_{i i}$ cannot be an eigenvalue of $A$, for some $i \geqslant 2$, since $A-a_{i i} I$ is always nonsingular (cf. the proof of [7, Theorem 3.1]).

Proposition 3.2. Let $T$ be a star. Then any noncentral main diagonal of entry $A$ in $\mathcal{S}(T)$ is not repeated more than twice if and only if the eigenvalues of $A$ are all simple. 
Proof. Let $A=\left(a_{i j}\right) \in \mathcal{S}(T)$ and suppose that $a_{i_{1} i_{1}}=a_{i_{k} i_{k}}$, with $1<i_{1}<i_{k} \leqslant n$ and $k=2, \ldots, s$. We are assuming that the central vertex is labeled by 1 . If $s \geqslant 3$, then $a_{i_{1} i_{1}}$ is an eigenvalue of $A$ with multiplicity at least 2 . If $s=2$, then $a_{i_{1} i_{1}}$ is an eigenvalue of $A$ ([7, Theorem 3.1]). Since the diagonal entry $a_{i_{1} i_{1}}$ is not an eigenvalue of $A\left(T \backslash\left\{1, i_{1}, j_{2}\right\}\right)$, from Lemma 2.4 , it is simple as an eigenvalue of $A$. If all noncentral main diagonal entries of $A$ are distinct, then $a_{i i}$ is not an eigenvalue $A$, for $i \geqslant 2$, from Lemma 3.1. Now, from (1), the maximum multiplicity of any eigenvalue of $A$ is 1 . This completes the proof.

Remark 3.3. Observe that no particular condition is imposed on the $(1,1)$-entry, i.e., the central main diagonal entry. In the case of the repetition of a noncentral diagonal main diagonal entry is greater than twice, that entry is an eigenvalue with multiplicity bigger than 1.

\section{The general case}

The previous section motivates a more general analysis of the distinctness of the diagonal entries of a matrix whose graph is $S_{n_{1} n_{2}}^{p}$ and the simplicity of its eigenvalues.

Proposition 4.1. Let A be a symmetric matrix whose graph is a double star, such that the noncentral main diagonal entries of each pendant star are distinct. Then the eigenvalues of $A$ are simple.

Proof. Let $A \in \mathcal{S}\left(S_{n_{1} n_{2}}^{p}\right)$, with $p>1$, and let $P$ be the (only) path joining the central vertices, say 1 and $n_{1}+p-1$, of the two pendant stars. Setting $V_{\bar{P}}=\left\{2, \ldots, n_{1}, n_{1}+p, \ldots, n_{1}+p+n_{2}-2\right\}$, for $V\left(S_{n_{1} n_{2}}^{p} \backslash P\right)$, with some natural conventions, we have

$$
\varphi\left(A\left(S_{n_{1} n_{2}}^{p} \backslash P\right), x\right)=\prod_{k \in V_{\bar{P}}}\left(x-a_{k k}\right) .
$$

From Lemma 2.4, if $\theta$ is an eigenvalue of $A$, then

$$
m_{A\left(S_{n_{1} n_{2}}^{p} \backslash P\right)}(\theta) \geqslant m_{A\left(S_{n_{1} n_{2}}^{p}\right)}(\theta)-1 .
$$

Therefore, the only eigenvalues with possible multiplicity greater than 1 are $a_{k k}$, for $k \in V_{\bar{P}}$.

Taking into account our assumptions, suppose that there are two pendant vertices, say $k_{1}$ and $k_{2}$, in the two pendant stars, such that $a_{k_{1} k_{1}}=a_{k_{2} k_{2}}$. Considering the (only) path $Q$ joining $k_{1}$ and $k_{2}$, and setting $V_{\bar{Q}}=V_{\bar{P}} \backslash\left\{k_{1}, k_{2}\right\}$, we have

$$
\varphi\left(A\left(S_{n_{1} n_{2}}^{p} \backslash Q\right), x\right)=\prod_{k \in V_{\bar{Q}}}\left(x-a_{k k}\right),
$$

and, thus, $a_{k_{1} k_{1}}$ is not a zero of $\varphi\left(A\left(S_{n_{1} n_{2}}^{p} \backslash Q\right), x\right)$, i.e.,

$$
m_{A\left(S_{n_{1} n_{2}}^{p} \backslash Q\right)}\left(a_{k_{1} k_{1}}\right)=0 .
$$

This means that

$$
m_{A\left(S_{n_{1} n_{2}}^{p}\right)}\left(a_{k_{1} k_{1}}\right) \leqslant 1 .
$$

The same procedure is applied in the case where a noncentral main diagonal entry $a_{k k}$ of one pendant star is not equal to any of the other pendant star. In this case, we consider $Q$ as joining $k$ to any pendant vertex of the other star.

We should point out that if some pendant entries of the same star are equal, Proposition 4.1 does not hold. For example, let us consider the double star $S_{32}^{4}$ : 


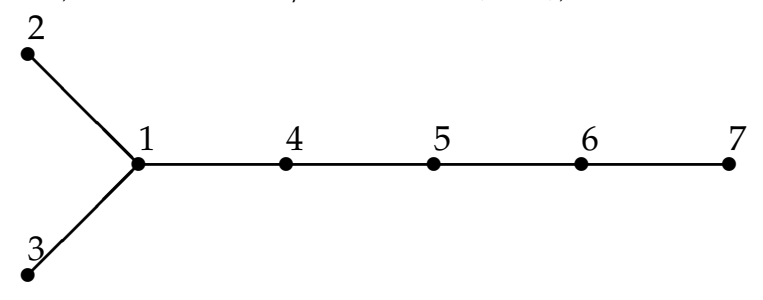

Figure 1: The graph $S_{32}^{4}\left(\right.$ or $\left.S_{30}^{5}\right)$.

and the matrix

$$
A=\left(\begin{array}{lllllll}
1 & 1 & 1 & 1 & 0 & 0 & 0 \\
1 & 1 & 0 & 0 & 0 & 0 & 0 \\
1 & 0 & 1 & 0 & 0 & 0 & 0 \\
1 & 0 & 0 & 1 & 1 & 0 & 0 \\
0 & 0 & 0 & 1 & 1 & 1 & 0 \\
0 & 0 & 0 & 0 & 1 & 2 & 1 \\
0 & 0 & 0 & 0 & 0 & 1 & 2
\end{array}\right)
$$

We observe that the entries $(2,2)$ and $(3,3)$ are equal to 1 , which is an eigenvalue of multiplicity 2 of $A$, and the vertices 2 and 3 belong to the same pendant star.

\section{The complete characterization}

For a given tree $T$, let us denote by $\mathcal{S D P}(T)$ the set of all matrices $A \in \mathcal{S}(T)$ whose graph is $T$, such that the noncentral main diagonal entries of each pendant star are distinct.

In Proposition 4.1 we proved that if $A \in \mathcal{S} \mathcal{D} \mathcal{P}\left(S_{n_{1} n_{2}}^{p}\right)$, all the eigenvalues of $A$ are simple. The converse is also true, i.e., if $A \in \mathcal{S D P}(T)$, for some tree $T$, then $T=S_{n_{1} n_{2}}^{p}$. Applying Lemma 1.3 in a more general and natural way, the proof of this claim goes along the same line as the necessity of Theorem 1.2 (cf. [16]). Obviously, the only part which may change is the clear construction of the matrix $D$.

Lemma 5.1. Let $T$ be a nontrivial tree. Then there exists a singular matrix in $\mathcal{S D P}(T)$ with all nonzero diagonal entries.

Therefore, we may establish the full characterization of the graphs of the matrices in $\mathcal{S D P}(T)$ :

Theorem 5.2. Let $T$ be a tree. Then each matrix in $\mathcal{S D P}(T)$ has distinct eigenvalues if and only if $T$ is a double star.

The following example presents a matrix whose graph is not a double star, with two distinct main diagonal entries indexed by pendant vertices, and having a nonsimple eigenvalue.

Let us consider the matrix

$$
A=\left(\begin{array}{lllllll}
2 & 2 & 2 & 2 & 0 & 0 & 0 \\
2 & 1 & 0 & 0 & 0 & 0 & 0 \\
2 & 0 & 3 & 0 & 0 & 0 & 0 \\
2 & 0 & 0 & 2 & 2 & 2 & 0 \\
0 & 0 & 0 & 2 & 1 & 0 & 0 \\
0 & 0 & 0 & 2 & 0 & 4 & 2 \\
0 & 0 & 0 & 0 & 0 & 2 & 4
\end{array}\right)
$$

whose graph $G$ is

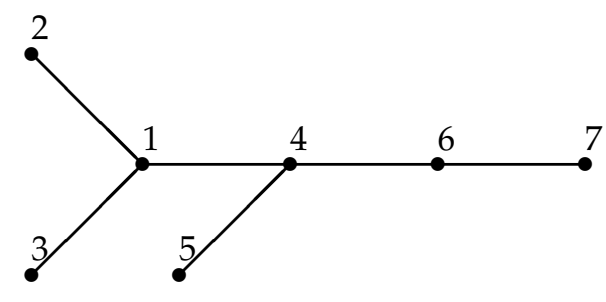


Clearly $G$ is not a double star and one can observe that on the one hand the vertices 2 and 3 belong to the same pendant star, and on the other hand the entries $(2,2)$ and $(3,3)$ are distinct, but 2 is an eigenvalue of multiplicity 2.

\section{Star complements}

In this section, we consider a second application of Lemma 2.3 which relies on the identification of the trees with a nonzero eigenvalue of maximum possible multiplicity due to Peter Rowlinson [19].

Let $\mu$ be an eigenvalue of multiplicity $m$ of a graph $G$. A set $X$ of $m$ vertices in $G$ such that $\mu$ is not an eigenvalue of $G-X$ is called a star set for $\mu$ in $G$ while the graph $G-X$ is called a star complement for $\mu$. Star sets and star complements exist for every eigenvalue of any graph. Originally, star sets were defined for the standard $(0,1)$-adjacency matrix of graph, but such notion can be extended to general weighted graphs, i.e., to any real symmetric matrix [3, Chapter 7]. For an update on the properties of star complements the reader is referred [4, Chapter 3].

Suppose that $\lambda_{1}, \ldots, \lambda_{m}$ are distinct eigenvalues of a graph $G$. We call $X_{1} \oplus \cdots \oplus X_{m}$ a star partition of (the set of vertices of) $G$ if $\lambda_{i}$ is not an eigenvalue of $G-X_{i}$, for $i=1, \ldots, m$.

Lemma 6.1. [2] If $S \subset X_{i}$, then $\lambda_{i}$ is an eigenvalue of $G-S$ with multiplicity $m_{G}\left(\lambda_{i}\right)-|S|$.

We remark that $m_{G}\left(\lambda_{i}\right)=\left|X_{i}\right|$.

Recently, Rowlinson added a new property [19, Lemma 1.1] to a star set for a graph in order to identify the trees with a nonzero eigenvalue of maximum possible multiplicity. The result is proved for a $(0,1)$ adjacency matrix of the graph. Here, as a consequence of Lemma 2.3, we provide a more detailed account of that property. First recall that a bridge of a connected graph is an edge whose removal disconnects the graph. In general, a bridge path is a path all of whose edges are bridges.

Proposition 6.2. Let $u, v$ be two distinct vertices in a star set for the weighted graph $G$, joined by a path $P_{u v}$ in the same star set. Then $P_{u v}$ is not a bridge path of $G$.

Proof. If $P_{u v}$ is a bridge path of $G$ contained in a star set $X$, associated to an eigenvalue $\lambda$, then, from Lemma 6.1, $m_{G-P_{u v}}(\lambda)=m_{G}(\lambda)-\left|P_{u v}\right|$. But, from Lemma 2.3, $m_{G-P_{u v}}(\lambda) \geqslant m_{G}(\lambda)-1$. Since $\left|P_{u v}\right| \geqslant 2$, we reach a contradiction.

In particular, if $G$ is a tree, each $X_{i}$ is a set of independent vertices. From Proposition 6.2 one can also easily derive the main tool used in [19].

Corollary 6.3 ([19]). If $u, v$ are adjacent vertices in a star set for $G$, then the edge $u v$ is not a bridge of $G$.

\section{Acknowledgements}

The authors are grateful to Milica Andjelić for many helpful discussions. We also thank the referee for the careful reading of the manuscript.

\section{References}

[1] D. Cvetković, M. Doob, H. Sachs, Spectra of Graphs. Theory and Applications, third edition, Johann Ambrosius Barth, Heidelberg, 1995.

[2] D. Cvetković, P. Rowlinson, S.K. Simić, A study of eigenspaces of graphs, Linear Algebra Appl. 182 (1993) 45-66.

[3] D. Cvetković, P. Rowlinson, S.K. Simić, Eigenspaces of graphs, Encyclopedia of Mathematics and its Applications, 66, Cambridge University Press, Cambridge, 1997.

[4] D. Cvetković, P. Rowlinson, S.K. Simić, Spectral generalizations of line graphs. On graphs with least eigenvalue -2 , London Mathematical Society Lecture Note Series, 314, Cambridge University Press, Cambridge, 2004.

[5] C.M. da Fonseca, A lower bound for the number of distinct eigenvalues of some symmetric matrices, Electron. J. Linear Algebra 21 (2010) 3-11. 
[6] C.M. da Fonseca, On the multiplicities of eigenvalues of a Hermitian matrix whose graph is a tree, Ann. Mat. Pura Appl. 187 (2008) 251-261.

[7] C.M. da Fonseca, On the inertia sets of some symmetric sign patterns, Czechoslovak Math. J. 56(131) (2006) 875-883.

[8] C.M. da Fonseca, A note on the multiplicities of the eigenvalues of a graph, Linear Multilinear Algebra 53 (2005) 303-307.

[9] C.M. da Fonseca, Interlacing properties for Hermitian matrices whose graph is a given tree, SIAM J. Matrix Anal. Appl. 27 (2005), no. $1,130-141$.

[10] C.D. Godsil, Algebraic matching theory, Electron. J. Combin. 2 (1995) \#R8.

[11] C.D. Godsil, Algebraic Combinatorics, Chapman and Hall, New York and London, 1993.

[12] C.D. Godsil, Spectra of trees, Ann. Discrete Math. 20 (1984) 151-159.

[13] C.R. Johnson, A. Leal Duarte, C.M. Saiago, The Parter-Wiener theorem: refinement and generalization, SIAM J. Matrix Anal. Appl. 25 (2003) 352-361.

[14] C.R. Johnson, R. Loewy, P.A. Smith, The graphs for which the maximum multiplicity of an eigenvalue is two, Linear Multilinear Algebra 57 (2009), no. 7, 713-736.

[15] C.R. Johnson, B.D. Sutton, Hermitian matrices, eigenvalue multiplicities, and eigenvector components, SIAM J. Matrix Anal. Appl. $26(2004 / 05)$ 390-399.

[16] I.-J. Kim, B.L. Shader, Classification of trees each of whose associated acyclic matrices with distinct diagonal entries has distinct eigenvalues, Bull. Korean Math. Soc. 45 (2008) 95-99.

[17] I.-J. Kim, B.L. Shader, On Fiedler- and Parter-vertices of acyclic matrices, Linear Algebra Appl. 428 (2008) 2601-2613.

[18] S. Parter, On the eigenvalues and eigenvectors of a class of matrices, J. Soc. Indust. Appl. Math. 8 (1960) 376-388.

[19] P. Rowlinson, On multiple eigenvalues of trees, Linear Algebra Appl. 432 (2010) 3007-3011.

[20] G. Szegő, Orthogonal Polynomials, Eleventh edition, American Mathematical Society Colloquium Publications, Vol. 23, American Mathematical Society, Providence, R.I., 2003.

[21] G. Wiener, Spectral multiplicity and splitting results for a class of qualitative matrices, Linear Algebra Appl. 61 (1984) 15-29. 